\title{
Impact of ICRH on the measurement of fusion alphas by collective Thomson scattering in ITER
}

Salewski, Mirko; Eriksson, L.-G.; Bindslev, Henrik; Korsholm, Søren Bang; Leipold, Frank; Meo, Fernando; Michelsen, Poul; Nielsen, Stefan Kragh

Published in:

Nuclear Fusion

Link to article, DOI:

$10.1088 / 0029-5515 / 49 / 2 / 025006$

Publication date:

2009

Link back to DTU Orbit

Citation $(A P A)$ :

Salewski, M., Eriksson, L-G., Bindslev, H., Korsholm, S. B., Leipold, F., Meo, F., Michelsen, P., \& Nielsen, S. K. (2009). Impact of ICRH on the measurement of fusion alphas by collective Thomson scattering in ITER. Nuclear Fusion, 49(2), 025006. https://doi.org/10.1088/0029-5515/49/2/025006

\section{General rights}

Copyright and moral rights for the publications made accessible in the public portal are retained by the authors and/or other copyright owners and it is a condition of accessing publications that users recognise and abide by the legal requirements associated with these rights.

- Users may download and print one copy of any publication from the public portal for the purpose of private study or research.

- You may not further distribute the material or use it for any profit-making activity or commercial gain

- You may freely distribute the URL identifying the publication in the public portal 


\title{
Impact of ICRH on the Measurement of Fusion Alphas by Collective Thomson Scattering in ITER
}

\author{
M Salewski ${ }^{1}$, L-G Eriksson ${ }^{2}$, H Bindslev ${ }^{1}$, S B Korsholm ${ }^{1}$, \\ F Leipold ${ }^{1}$, F Meo ${ }^{1}$, P K Michelsen ${ }^{1}$, and S K Nielsen ${ }^{1}$ \\ ${ }^{1}$ Association Euratom - Risø National Laboratory for Sustainable Energy, Technical \\ University of Denmark, DK-4000 Roskilde, Denmark \\ ${ }^{2}$ CEA, IRFM, F-13108 St. Paul lez Durance, France \\ E-mail: msal@risoe.dtu.dk
}

\begin{abstract}
Collective Thomson scattering (CTS) has been proposed for measuring the phase space distributions of confined fast ion populations in ITER plasmas. This study determines the impact of fast ions accelerated by ion cyclotron resonance heating (ICRH) on the ability of CTS to diagnose fusion alphas in ITER. Fast ions with large perpendicular velocities, such as the populations investigated here, can be detected with the "enabled" part of the proposed ITER CTS diagnostic. The investigated ICRH scenarios include pure second harmonic tritium heating and ${ }^{3} \mathrm{He}$ minority heating at a frequency of $50 \mathrm{MHz}$, corresponding to an off-axis resonance. The sensitivities of the results to the ${ }^{3} \mathrm{He}$ concentration $(0.1-4 \%)$ and the heating power $(20-40 \mathrm{MW})$ are considered. Fusion born alphas dominate the total CTS signal for large Doppler shifts of the scattered radiation. The tritons generate a negligible fraction of the total fast ion CTS signal in any of these heating scenarios. The minority species ${ }^{3} \mathrm{He}$, however, contributes more than $10 \%$ of the fast ion CTS signal at locations close to the resonance layer for ${ }^{3} \mathrm{He}$ concentrations larger than $\sim 1 \%$. In this particular region in space for resolution of near perpendicular velocities, it may be difficult to draw conclusions about physics of alpha particles alone by CTS. With this exception, the CTS diagnostic can reveal the physics of the fusion alphas in ITER even under presence of fast ions due to ICRH.
\end{abstract}

PACS numbers: 52.25.Os, 52.40.Db, 52.50.Gj, 52.50.Qt, 52.65.Cc, 52.70.Gw

\section{Introduction}

Burning plasmas such as foreseen in ITER inherently contain large populations of ions with supra-thermal energies. Fast ions in present plasma confinement devices are mainly produced by auxiliary heating methods: Ion cyclotron resonance heating (ICRH) and neutral beam injection (NBI). In ITER, however, the dominant source of fast ions and heating will be the deuterium - tritium fusion reaction producing energetic alpha particles $[1,2]$. These fast ion populations with energies up to several $\mathrm{MeV}$ provide the means to heat the plasma and thereby balance the energy losses. In burning plasmas, the fast ions need to be well confined while they slow down and transfer their energy 
to the thermal plasma as the fast ion confinement has direct impacts on the achievable heating efficiency. Additionally, the losses of energetic ions to the wall need to be limited to avoid unacceptable heat loads on the wall, especially in view of the very long pulse times and large energetic ion populations planned for ITER [3]. In burning plasmas, therefore, fast ions play an even more important role than in current plasmas.

Fast ion losses due to single-particle effects are relatively well understood, but fast ion losses due to collective behaviour are much more challenging. Among these are the normal modes such as the family of Alfvén gap modes, kinetic ballooning modes, and internal kink modes $[4,5]$. Perhaps the most serious of these in ITER is the toroidal Alfvén eigenmode [6-10]. The velocities of fast ions in ITER are comparable to the Alfvén speed, and fast ions can therefore resonantly excite shear Alfvén waves which are weakly damped in gaps in the shear Alfvén continuum. Also of importance in a burning plasma may be the branch of energetic particle modes for which the drive can overcome continuum damping if the fast ion pressure is above a threshold [11,12]. These classes of modes may redistribute and eject fast ions, and their interaction with the less anisotropic fast ion populations of burning plasmas with a higher degree of self-organization is still not accurately known. The radial transport of fast ions may be large due to these effects.

The measurement of phase space distributions of confined fast ions is all the more important for understanding these energetic ion physics issues in the burning plasma regime. Measurement of fast ions in ITER is essential for benchmarking the predictions made by current theories. Collective Thomson scattering (CTS) is a multi-facetted diagnostic with which the 1D fast ion velocity distribution function in plasmas can be determined. This has been demonstrated at JET and TEXTOR [13-16]. CTS at ASDEX Upgrade is in the commissioning phase [17].

ICRH accelerates ions to large velocities perpendicular to the magnetic field, leading to strongly anisotropic fast ion distributions. In burning plasmas, there is additionally the approximately isotropic population of fusion alphas. The fast ions due to ICRH may affect the CTS alpha measurements near the perpendicular direction (see Section 2): It is not feasible to tell from the measured CTS signal how large the contributions of the various fast ion species to the measured signal are since the experimentally accessible quantity is the sum of all contributions. For example, an alpha produces as much CTS signal as four tritons moving at the same velocity. Nevertheless, their fractions of the total CTS signal can be found by modelling $[18,19]$. This is performed here in a series of synthetic diagnostic experiments with the goal to determine which species will dominate the signal for the planned ITER CTS diagnostic resolving near perpendicular velocities and will therefore be amenable to direct observation to a good approximation.

In previous studies, it was found that beam ions from NBI heating in ITER create a small protrusion in the CTS spectrum of the near parallel velocity component for frequency upshifts within a limited frequency band in a limited region in space [20,21]. However, the beam ion fraction of the CTS signal is negligible for frequency downshifts concordant with the fact that NBI produces highly directed fast ion distributions. It 
could be concluded that the beam ions will not significantly affect the measurement of alpha particles. As explained in Section 2, the measurement of near parallel velocity components requires a receiver on the high field side (HFS) which is not part of the present ITER design. Near perpendicular velocities, on the other hand, can be measured with a receiver on the low field side (LFS) which fits into an ITER port plug. This configuration has been "enabled" as ITER diagnostic [22]. The present study focusses on this "enabled" system considering various ICRH scenarios and thereby complements the previous studies of NBI heating.

The distribution functions for resonant tritons and ${ }^{3} \mathrm{He}$ (if present) have been computed with the PION code $[23,24]$ and the resulting contributions to the CTS signal with a fully electromagnetic model of CTS $[18,19]$. The assumed heating scenarios were pure tritium heating at the second harmonic resonance and ${ }^{3} \mathrm{He}$ minority heating at the fundamental resonance with ${ }^{3} \mathrm{He}$ concentrations from $0.1 \%$ to $4 \%$. The ICRH frequency was set to $50 \mathrm{MHz}$, corresponding to an off-axis resonance on the LFS. The heating power was varied from 20 to $40 \mathrm{MW}$.

In Section 2, the ITER CTS system and the models describing it are discussed. Modelling of the plasma parameters, among these the fast ion distributions, is described in Section 3. Section 4 contains the computed fast ion distributions and corresponding CTS spectra for resolution of near perpendicular velocities in the heating scenarios mentioned above, and conclusions are drawn in Section 5. The results indicate that the CTS signal in the frequency bands of interest will mostly originate from fusion born alpha particles. Fast tritons produce a negligible fraction of the CTS signal compared to fusion alphas in any investigated heating scenario. However, if the minority species ${ }^{3} \mathrm{He}$ is present in concentrations larger than $1 \%$, it generates a significant fraction of the CTS signal $(>10 \%)$ in the outer frequency bands typical for fast ions at locations close to the resonance layer.

\section{Collective Thomson Scattering Modelling and Design for ITER}

Microscopic fluctuations in the plasma will scatter radiation from a beam of radiation passing through the plasma. The ion velocity distribution can be inferred from the microscopic fluctuations with a wavelength larger than the Debye length $\lambda_{D}$, i.e. $k^{\delta} \lambda_{D}<1$, where $k^{\delta}$ is the magnitude of the fluctuation wave vector $\mathbf{k}^{\delta}$. In the experiment, a beam of probing radiation with wavenumber $\mathbf{k}^{i}$ is launched into the plasma, and part of the scattered radiation with wavenumber $\mathbf{k}^{s}$ is detected by a receiver. The measurement is spatially localized in the so-called scattering volume which is given by the overlap of probe and receiver beams. The receiver beam is an imagined beam which would emerge if one followed the path of radiation accepted by the receiver in reverse direction. Example probe and receiver beams and scattering volumes in ITER are sketched in Figure 1. The wavenumbers and frequencies (with identical superscripts) are related by the conservation principles $\left(\mathbf{k}^{\delta}, \omega^{\delta}\right)=\left(\mathbf{k}^{s}-\mathbf{k}^{i}, \omega^{s}-\omega^{i}\right)$. The fluctuation frequency $\omega^{\delta}$ driven by a fast ion can be approximately related to the ion velocity by 


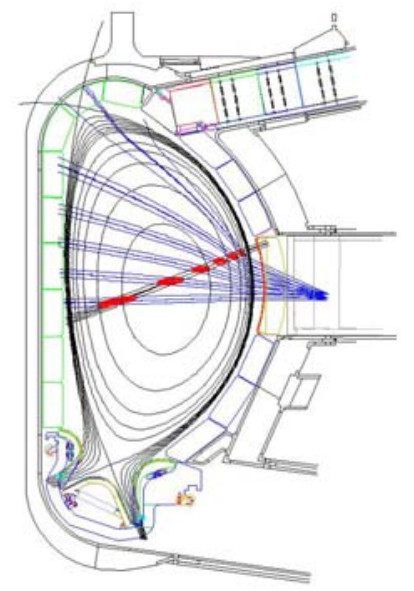

(a) ITER poloidal plane with a probe and several receiver beams

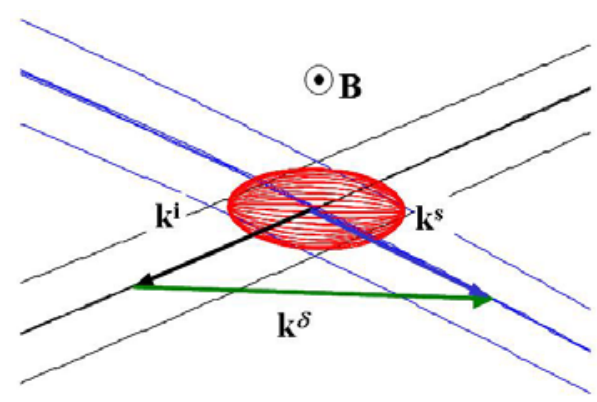

(b) Scattering geometry with overlap of probe and receiver beams, making up the scattering volume

Figure 1. Sketch of the CTS subsystem for ITER showing resolution near perpendicular to the magnetic field

$\omega^{\delta}=\mathbf{v}_{\text {ion }} \cdot \mathbf{k}^{\delta}$. This corresponds to resolution of the projection $g$ of the full velocity distribution function $f$ along the direction of $\mathbf{k}^{\delta}$ in the scattering volume, expressed by ( $\delta$ is here the Dirac $\delta$-function): $g(u)=\int d^{3} \mathbf{v} f \delta\left(\frac{\mathbf{v} \cdot \mathbf{k}^{\delta}}{k^{\delta}}-u\right)$. The measurable quantity in CTS is the spectral power density of scattered radiation $\frac{\partial P^{s}}{\partial \omega^{s}}$ which is proportional to the scattering function $\Sigma$. The scattering function accounts for the spectral variation in the scattered radiation due to microscopic fluctuations in the plasma. It is considered in a fully electromagnetic model and depends on fluctuations in electron density $\tilde{n}$, the electric field $\tilde{\mathbf{E}}$, the magnetic field $\tilde{\mathbf{B}}$, and the current $\tilde{\mathbf{j}}[18,19]$. We present the results in this study in terms of the scattering function.

The proposed ITER CTS system is designed to measure time-resolved fast ion velocity distributions in several measurement volumes simultaneously, satisfying the ITER measurement requirements for fusion alpha diagnostic [25]. It is divided into two subsystems, one for measuring fast ion velocity distributions in near perpendicular directions and one for near parallel directions [21,26-29]. Each system has its own launcher which couples a probe beam of electromagnetic waves at $60 \mathrm{GHz}$ in X-mode into the plasma on the LFS. The probe sources are $1 \mathrm{MW}$ gyrotrons. For resolution of near parallel velocities, a receiver on the HFS is required while a receiver on the LFS is needed for resolution of near perpendicular velocities. The system we focus on here is the one with the receiver antenna on the LFS which has recently been "enabled" as ITER diagnostic. This system is sketched in Figure 1(a). Contrarily, the receiver on the HFS is not part of the current ITER design. The location of the scattering volume is described here by coordinates $R$ (distance from the torus center) and $Z$ (height above the 
plane which contains the magnetic axis). Two angles are most relevant when describing the scattering geometry: The angle $\phi$ between the magnetic field vector $\mathbf{B}$ and $\mathbf{k}^{\delta}$ and the scattering angle $\theta$ between the probe and receiver beams.

\section{Modelling of Plasma Parameters}

The profiles of the bulk ion species, impurities, and the electrons at the respective measurement location are assumed to be given by the steady-state ITER plasma equilibrium [30]. The parameters for this ITER scenario were taken from simulations with the ASTRA code [31]. We assume bulk plasma species to have Maxwellian distribution functions and the fusion alphas to have an isotropic classical slowing down distribution. The $4 \mathrm{D}$ distribution functions $\left(R, Z, v_{\|}, v_{\perp}\right)$ of the fast ion populations from ICRH have been calculated with the PION code [23, 24]. In the simulations for the CTS diagnostic presented here, it is important to assess the pitch angle dependence of the distribution functions of the resonating ions. For this purpose, a model for the pitch angle distribution in the small banana width limit, which is similar to that found in e.g. Ref. [32], has been added to the standard PION code [21]. This model should provide acceptable results in cases for which finite orbit width effects are not expected to be important. It is important to note that the simulations presented here do not include self-consistent coupling between the plasma parameter profiles and the auxiliary heating which would require large development efforts. Additionally, information about the hardware details of the ICRH system is still not available.

\section{Results}

\subsection{Pure Second Harmonic Tritium Heating}

ICRH scenarios without presence of the minority species ${ }^{3} \mathrm{He}$ are discussed in this section. The standard ITER reference design relies on second harmonic tritium heating with a power level of $20 \mathrm{MW}$. We investigate additionally an upgraded ICRH power of $40 \mathrm{MW}$ which has been under consideration and serves here as a sensitivity study. To maximize the ICRH power coupling to the plasma, the wave-particle resonance position should be located in a region of sufficiently high plasma density. For bulk ion heating it is advantageous to move the cyclotron resonance somewhat to the LFS [33]. The scenarios considered here therefore have the nominal magnetic field on axis, $5.3 \mathrm{~T}$, and an ICRH frequency of $50 \mathrm{MHz}$, placing the second harmonic cyclotron resonance of tritons (and the fundamental resonance of ${ }^{3} \mathrm{He}$ ions) at around a third of the minor radius on the LFS of the magnetic axis. The magnetic axis for this scenario is located at about $R_{0}=6.35 \mathrm{~m}$, and the resonance at about $R=6.9 \mathrm{~m}$. In this case, PION simulations suggest that finite orbit width effects only play a minor role.

Figure 2(a) provides an overview of the 2D velocity distribution function of tritium at $R=6.85 \mathrm{~m}$ and $Z=0.77 \mathrm{~m}$ for the scenario with $40 \mathrm{MW}$ ICRH. This location of the scattering volume leads to the strongest triton CTS signal component compared to 


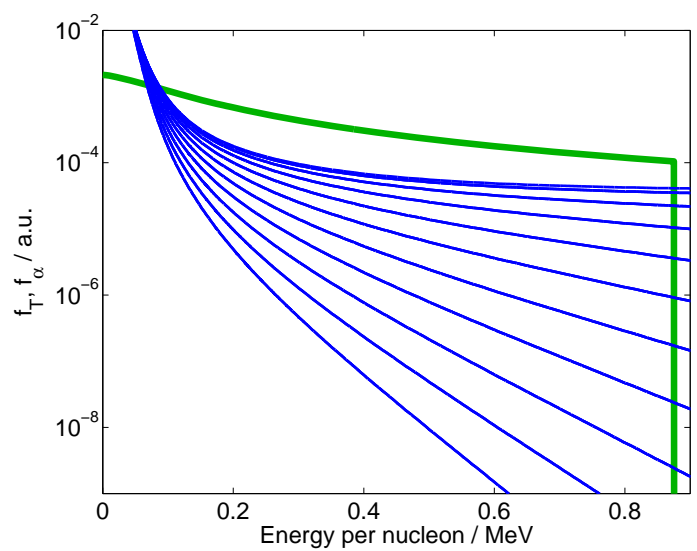

(a) Tritium distribution function compared to a classical slowing down distribution for alphas; thick green line - alphas; thin blue lines - Tritium at various equally spaced pitch angles from $0^{\circ}$ (bottom curve) to $90^{\circ}$ (top curve)

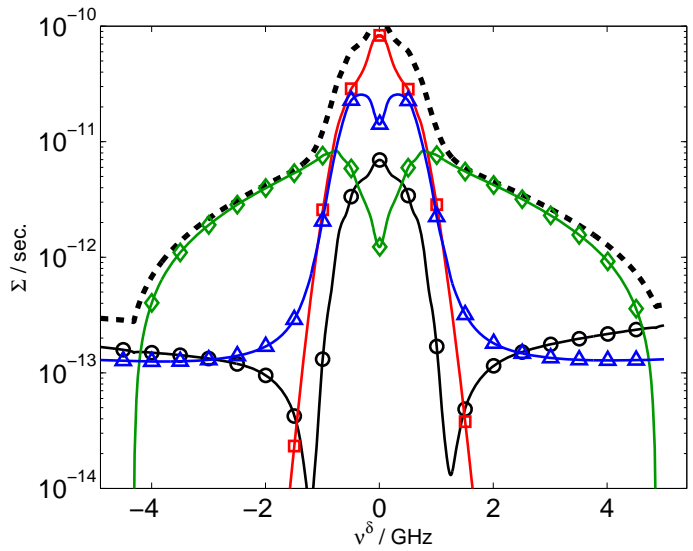

(b) Scattering function; scattering parameters: $\phi=101^{\circ}, \theta=156^{\circ}$; $\diamond$-alphas, $\triangle$-tritons, $\square$-bulk ions, $\bigcirc$ electrons, - - - total

Figure 2. Tritium distribution function and resulting CTS scattering function for pure second harmonic tritium heating with $40 \mathrm{MW}$ ICRH power at $R=6.85 \mathrm{~m}$ and $Z=0.77 \mathrm{~m}$

other locations. The energy distribution of tritium is plotted for various pitch angles together with a classical slowing down distribution for fusion alphas. The energy has been normalized by the atomic mass number such that the abscissa is proportional to the square of the ion speed. It is evident that the triton distributions are strongly anisotropic with large perpendicular velocities and small parallel velocities. Energy absorption in ICRH at the second harmonic increases with the Larmor radius, and tritons are hence accelerated to very high energies in the perpendicular direction (up to a point where the Larmor radius starts to become comparable to the perpendicular wavelength). The population of resonating ions with large parallel velocities is so small that it is of no concern in the context of the present study, even in a scenario with $40 \mathrm{MW}$ ICRH power. Therefore, the attention is focussed on the near perpendicular velocities in this study, measurable with the scattering geometries presented in Figure 1.

Resonating ions from ICRH can typically be found in a rather narrow region. In this work, the configuration space is scanned in small steps $(\sim 2-3 \mathrm{~cm})$ to find this region: The strongest CTS signal contribution from tritium is found at $R=6.85 \mathrm{~m}$ and $Z=0.77 \mathrm{~m}$ as mentioned above. The scattering function for this geometry is presented in Figure 2(b) for a power of $40 \mathrm{MW}$. The total signal for each frequency shift $\nu^{\delta}$ is the sum of the individual components. The fusion alphas dominate the spectrum for frequencies from $\sim \pm 1-4 \mathrm{GHz}$, the outermost tips of the wings (high frequency shift) corresponding to alpha birth velocities and the proximal ends of the wings (low 


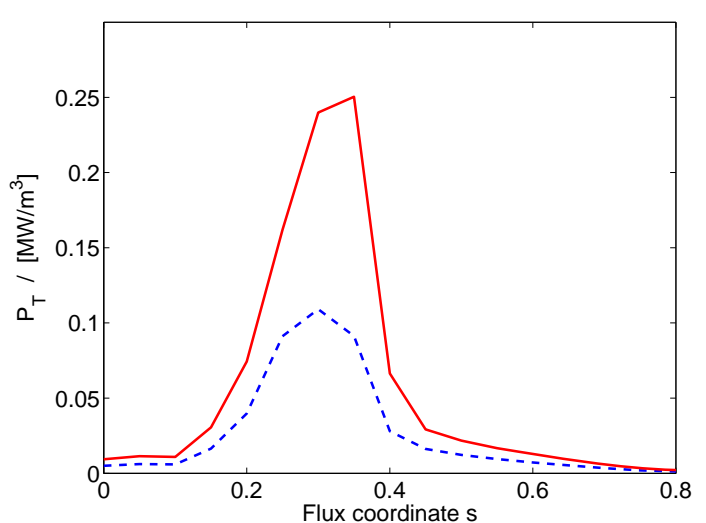

(a) Power deposition profile; - $40 \mathrm{MW}$, - - - $20 \mathrm{MW}$

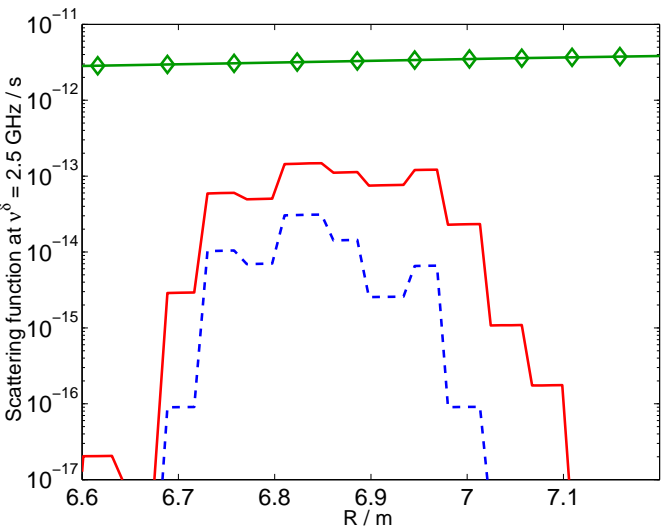

(b) Scattering function at $\nu^{\delta}=2.5 \mathrm{GHz}$ for various scattering volumes with different $R ; \diamond$ - alphas, - tritons at $40 \mathrm{MW}$, - - - tritons at $20 \mathrm{MW}$

Figure 3. Power deposition profile and profile of the scattering function at $\nu^{\delta}=$ $2.5 \mathrm{GHz}$ for pure second harmonic tritium heating

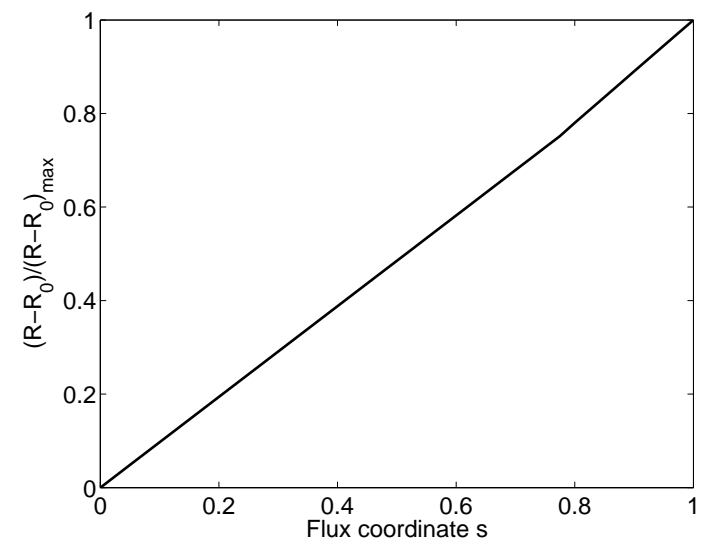

Figure 4. Mapping between the flux coordinate s and the normalized radial coordinate in the outer mid-plane

frequency shift) to thermalized alpha ash. At even larger Doppler shifts, the electrons generate the only significant feature in the spectrum, and the bulk ion CTS signal towers over the other species at smaller Doppler shifts $(<\sim \pm 1 \mathrm{GHz})$. The bulk ions contain deuterium and the impurities argon and beryllium. The tritons are singled out from the bulk (also from the thermal part) due to their highly energetic tail. Even for $40 \mathrm{MW}$ ICRH power, twice the ICRH power currently planned for ITER, the contribution of the tritons to the total CTS signal with large Doppler shifts will be at least an order of magnitude below the alpha contribution and roughly level with the electron contribution. Part of the reason for the much weaker triton CTS signal component compared to the alpha component is that CTS signals are proportional to the square of the ion charge. 
Additionally, the volumetric heating rates are relatively moderate for second harmonic tritium heating as Figure 3(a) shows: The power deposition profiles are plotted as a function of the flux coordinate $s$ which is the square root of the poloidal flux. The flux coordinate $s$ is zero on the magnetic axis and one at the plasma edge. The mapping between the flux coordinate $s$ and the minor radius in the outer midplane for the present equilibrium follows a nearly linear relation and is displayed in Figure 4. The power deposition profile on the tritium for second harmonic tritium heating may be compared to the power deposition profiles on ${ }^{3} \mathrm{He}$ for ${ }^{3} \mathrm{He}$ minority heating in Figure 7 . The discussion of this comparison is deferred to Section 4.2. The ICRH power is mainly deposited between $s=0.2$ and $s=0.4$. The $40 \mathrm{MW}$ scenario leads to a higher peak in the power deposition profile compared to the $20 \mathrm{MW}$ scenario. In Figure 3(b), one representative frequency shift $(+2.5 \mathrm{GHz})$ is plotted as a function of the major radius of the scattering volume for the reference power (20 MW) and the upgraded power (40 MW). The fusion alpha component is not affected by the two heating scenarios. If the ICRH power is increased, the triton CTS signal fraction will also increase as a result of the larger population of tritons at that frequency shift. The CTS signal component due to tritium in Figure $3(\mathrm{~b})$ is strongest in a region with a width of about $0.2-0.3 \mathrm{~m}$ located at a position around $R=6.85 \mathrm{~m}$ (the maximum) which was used for Figure 2 . This width is comparable to the width of scattering volumes $(\sim 0.2 \mathrm{~m})$. The spatial variation in the CTS signal component strength may be important due to this similarity in scale. In the modelling, a constant CTS signal throughout the scattering volume is assumed, leading to an overestimation at the maximum if the signal is non-uniform in configuration space (as for the clearly peaked tritium component). These considerations indicate that for ICRH of tritium at the second harmonic resonance (50 MHz), most of the fast ion CTS signal can be attributed to fusion alphas. This conclusion is also true for the tritium populations in ${ }^{3} \mathrm{He}$ minority heating scenarios to be discussed next: The triton CTS signal component always falls clearly short of the fusion alpha CTS signal component by more than an order of magnitude.

\section{2. ${ }^{3}$ He minority heating}

${ }^{3} \mathrm{He}$ minority heating is an interesting option for ICRH since it increases the amount of power coupled into the ions rather than the electrons. In contrast to the second harmonic heating scheme, fundamental minority heating does not depend on the perpendicular velocity of the resonating ions to lowest order in a finite Larmor radius (FLR) expansion. The minority ions therefore tend to be accelerated more uniformly in velocity space than in the case of ions accelerated by second harmonic interaction which is an FLR effect, i.e. the interaction becomes more efficient as the perpendicular velocity of a resonating ion increases (up to the limit mentioned above). Thus, in the case of a second harmonic interaction a smaller fraction of the resonating ions (those with high perpendicular velocity) are accelerated efficiently by the ICRH waves. The result is a tail on the distribution function of the resonating ions with on average more energetic ions, but at 


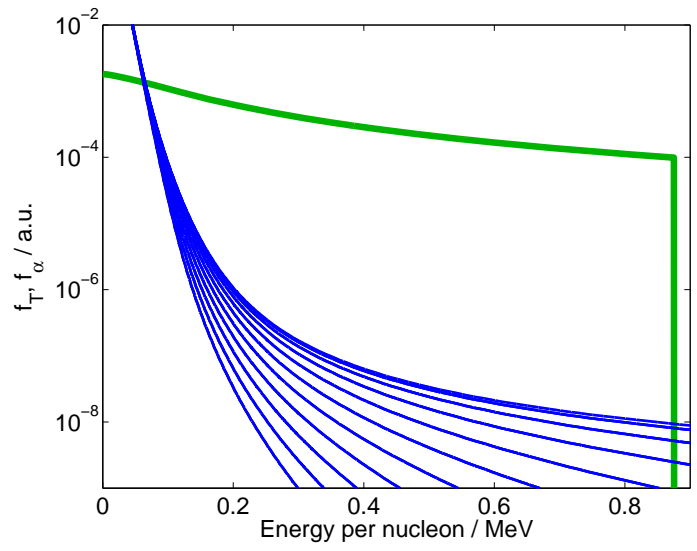

(a) Tritium

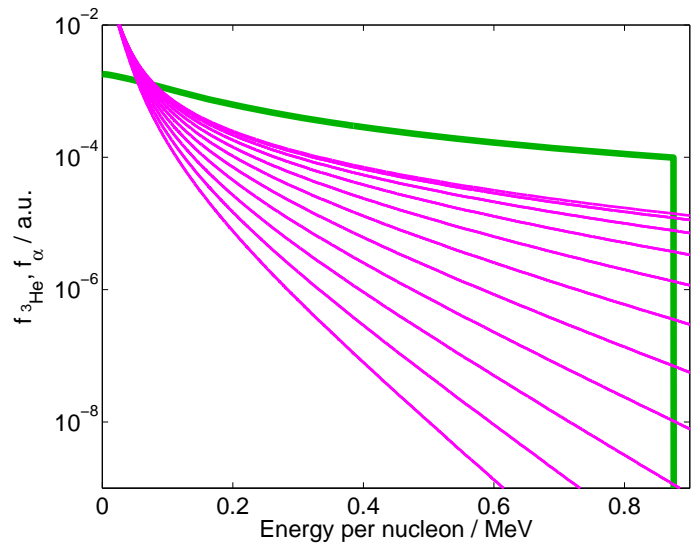

(b) ${ }^{3} \mathrm{He}$

Figure 5. Tritium and ${ }^{3} \mathrm{He}$ distribution functions for minority heating with $4 \%{ }^{3} \mathrm{He}$ for $R=6.76 \mathrm{~m}$ and $Z=0.76 \mathrm{~m}$ compared to a classical slowing down distribution for alphas; thick green line - alphas; thin blue lines in (a) - tritium; thin magenta lines in (b) $-{ }^{3} \mathrm{He}$; the thin lines show various equally spaced pitch angles from $0^{\circ}$ (bottom curve) to $90^{\circ}$ (top curve); the corresponding CTS signal is shown in Figure 6(b).

the same time their fraction is lower. Minority heating does not depend strongly on FLR effects, but an energetic tail forms since the energy per resonating particle is large for the minority species. ${ }^{3} \mathrm{He}$ minority heating may be a good choice during the start-up phase of a burning plasma but may be disadvantageous when the fusion alphas provide a large part of the heating as the ${ }^{3} \mathrm{He}$ dilutes the fuel. However, if minority heating is applied, the ${ }^{3} \mathrm{He}$ population will also be confined and therefore its concentration in the burning plasma may not drop very fast, leaving a small population of ${ }^{3} \mathrm{He}$. Moreover, radioactive decay of tritium leads to a ${ }^{3} \mathrm{He}$ nucleus, and hence deuterium-tritium plasmas will always contain at least trace amounts of ${ }^{3} \mathrm{He}$. We investigate here ${ }^{3} \mathrm{He}$ concentrations of $1-4 \%$ in $1 \%$ steps and also calculate a scenario with $0.1 \%{ }^{3} \mathrm{He}$ concentration.

The simulations indicate that the CTS signal fraction of ${ }^{3} \mathrm{He}$ as a function of ${ }^{3} \mathrm{He}$ concentration has a maximum in each case: $3 \%$ for $20 \mathrm{MW}$ and $4 \%$ or more for $40 \mathrm{MW}$ ICRH power. A concentration with maximum CTS signal exists due to the fact that a too low ${ }^{3} \mathrm{He}$ concentration obviously results in a very small fast ion population whereas a too high concentration leads to a low power per resonating particle. The maximum for minority heating with ${ }^{3} \mathrm{He}$ concentration of $4 \%$ and $40 \mathrm{MW}$ ICRH power lies at $R=6.76 \mathrm{~m}$ and $Z=0.76 \mathrm{~m}$. The $2 \mathrm{D}$ distribution functions of tritium and ${ }^{3} \mathrm{He}$ for this location are shown in Figure 5. The population of fast ${ }^{3} \mathrm{He}$ is evidently much larger than the triton population at this location.

The scattering functions for $3 \%{ }^{3} \mathrm{He}$ with $20 \mathrm{MW}$ and $4 \%{ }^{3} \mathrm{He}$ with $40 \mathrm{MW}$ are revealed in Figures 6(a) and 6(b), respectively. The latter corresponds to the 2D distribution function shown in Figure 5. It becomes clear that the ${ }^{3} \mathrm{He}$ for minority heating produces a stronger signal contribution than the tritium does for pure tritium 


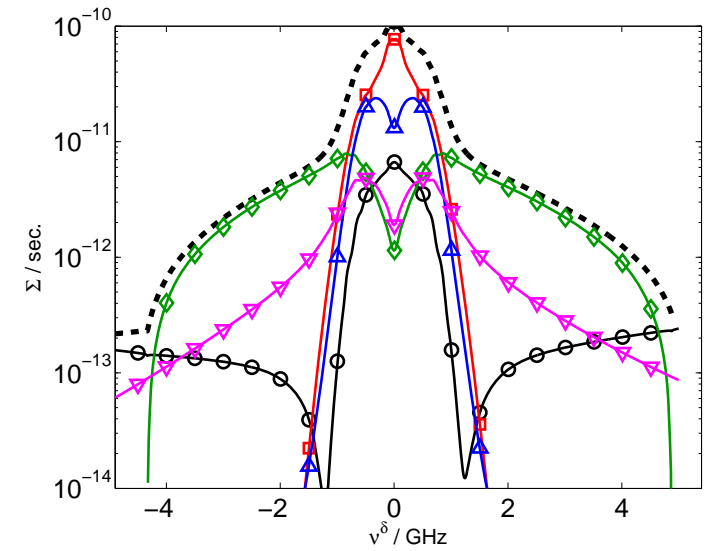

(a) $3 \%{ }^{3} \mathrm{He}$ concentration and $20 \mathrm{MW}$ heating power

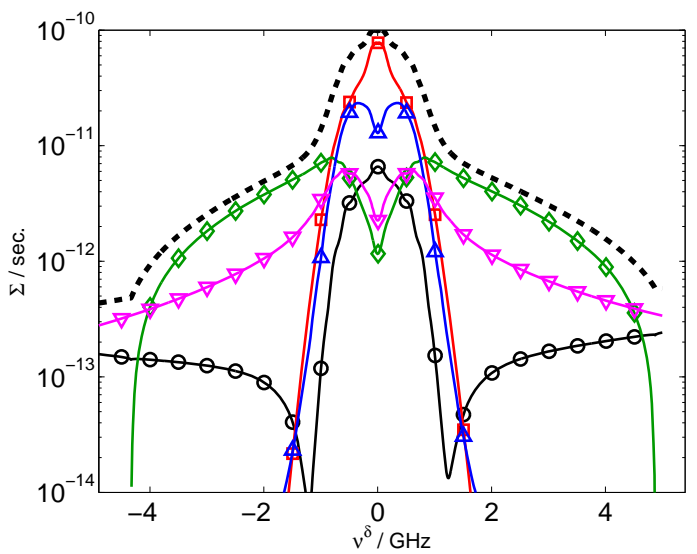

(b) $4 \%{ }^{3} \mathrm{He}$ concentration and $40 \mathrm{MW}$ heating power

Figure 6. Scattering functions resolving near perpendicular velocities; scattering parameters: $R=6.76 \mathrm{~m}, Z=0.76 \mathrm{~m}, \phi=101^{\circ}, \theta=157^{\circ} ; \diamond$-alphas, $\triangle$-tritons, $\nabla^{-}{ }^{3} \mathrm{He}, \square$-bulk ions, $\mathrm{O}$-electrons, - - - - total

heating. Contrary to the former case, the ${ }^{3} \mathrm{He}$ feature reaches up to $10-20 \%$ of the alpha feature even for the nominal ICRH power of $20 \mathrm{MW}$. The reason for the strong CTS signal component lies partly in the power deposition profiles and partly in the dependence of the CTS signal on the square of the charge of the ion species as mentioned in Section 4.1. The scattering volume with the maximum CTS signal contribution lies slightly towards the HFS for ${ }^{3} \mathrm{He}(R=6.76 \mathrm{~m})$ compared to tritium $(R=6.85 \mathrm{~m})$. The fundamental resonance of ${ }^{3} \mathrm{He}$ coincides with the second harmonic resonance of tritium and is located at about $R=6.9 \mathrm{~m}$ which is on the LFS of the magnetic axis $\left(R_{0}=6.35 \mathrm{~m}\right)$. However, in the minority heating scheme, the $E_{+}$component of the wave electric field (the left hand polarized component rotating in the Larmor direction of the resonating ions) is the primary source of acceleration of the minority species to lowest order in a Larmor radius expansion. The $E_{+}$component peaks sharply on the HFS of the cyclotron resonance, and ions seeing a Doppler broadened resonance on the HFS therefore have the strongest absorption. On the other hand, for second harmonic heating schemes there is no such strong variation of the $E_{+}$component near the cyclotron resonance. Thus, the ${ }^{3} \mathrm{He}$ cases have a maximum power absorption shifted towards the HFS as compared to pure second harmonic tritium heating. In the case of a resonance on the LFS of the magnetic axis, such a shift means that the power is absorbed in a smaller volume, fewer ions therefore absorb the power, making them on average more energetic. It becomes clear that only the minority species ${ }^{3} \mathrm{He}$ can produce a CTS signal contribution on the same order as the fusion alphas for ICRH, although it is still smaller and very localized.

The sensitivities of the power deposition profiles to the ${ }^{3} \mathrm{He}$ concentration and the ICRH power levels are displayed in Figures 7(a) and 7(b) for 20 and $40 \mathrm{MW}$, respectively. 


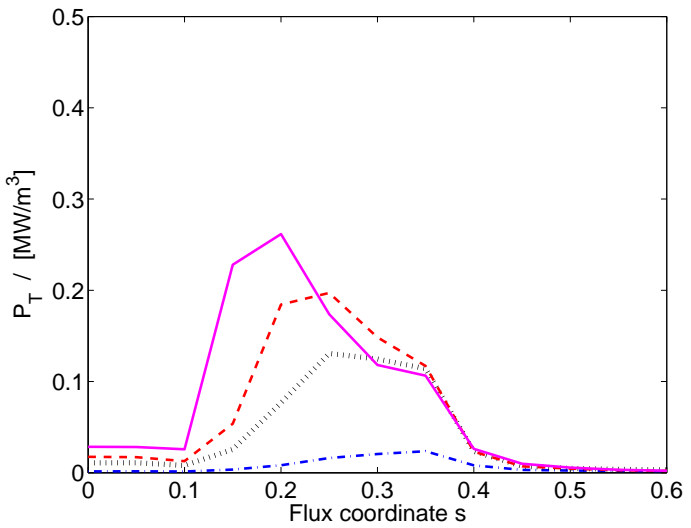

(a) 20 MW heating power

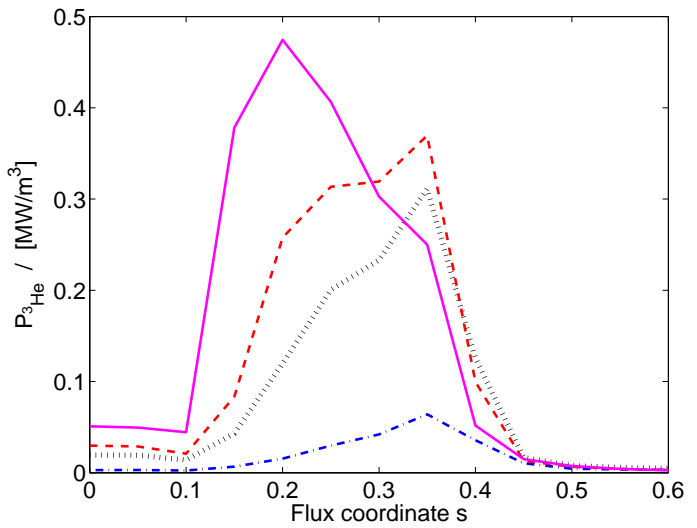

(b) $40 \mathrm{MW}$ heating power

Figure 7. Power deposition profiles on ${ }^{3} \mathrm{He}$ for various ${ }^{3} \mathrm{He}$ concentrations; $-4 \%$, - - - $2 \%, \cdots \cdots 1 \%,-.-0.1 \%$

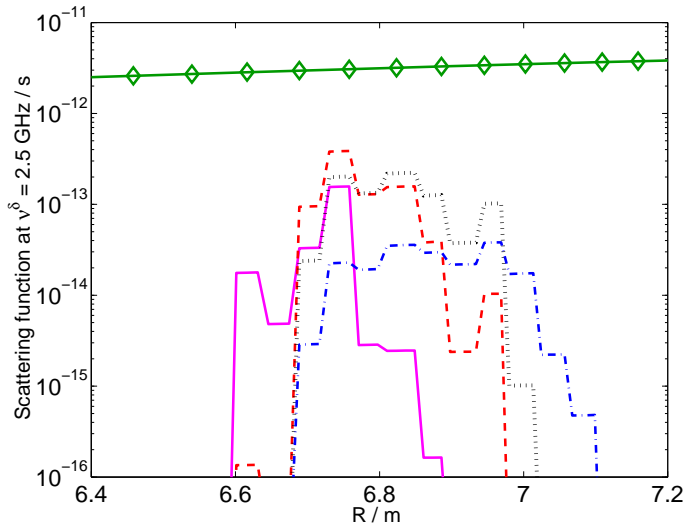

(a) 20 MW heating power

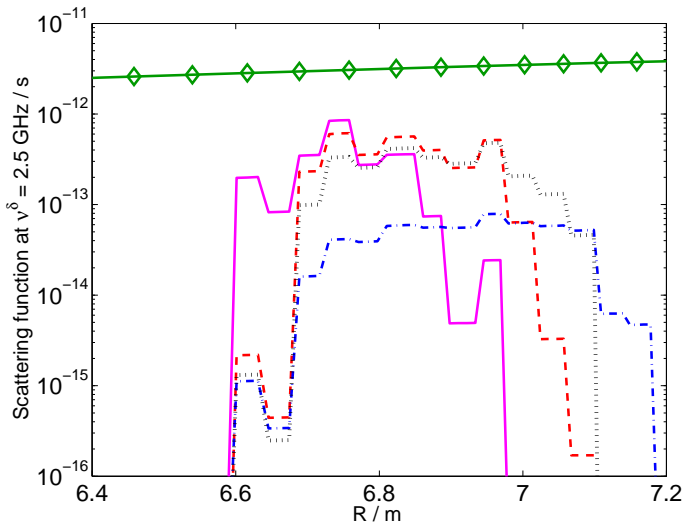

(b) $40 \mathrm{MW}$ heating power

Figure 8. Scattering function at $\nu^{\delta}=2.5 \mathrm{GHz}$ for various scattering volumes with different $R$ for various ${ }^{3} \mathrm{He}$ concentrations; $\diamond$-alphas, ${ }^{3} \mathrm{He}$ for various concentrations: $4 \%,-\ldots 2 \%, \cdots \cdots 1 \%,-\cdots-1 \%$

The results depend rather strongly on the ${ }^{3} \mathrm{He}$ concentration since the polarization of the wave and the absorption strength both change with ${ }^{3} \mathrm{He}$ concentration. For these low ${ }^{3} \mathrm{He}$ concentrations, the wave damping increases with concentration. Strong wave damping, occurring for large concentrations, leads to a very peaked deposition profile whereas lower damping leads to a less peaked deposition profile. The polarization affects the location of maximum power deposition, such that the power is deposited further towards the HFS for larger minority species concentrations. The volumetric effect then additionally leads to the larger power densities. In Figures 8(a) and 8(b), the scattering functions in this scenario are plotted for one representative frequency 
shift at $+2.5 \mathrm{GHz}$ as function of the major radius of the scattering volume. It may be noted that maximum power deposition does not necessarily lead to large populations of fast ${ }^{3} \mathrm{He}$. For example, the power deposition profile has even for $20 \mathrm{MW}$ the largest peak for $4 \%{ }^{3} \mathrm{He}$ concentration, but a larger population of fast ${ }^{3} \mathrm{He}$ develops for $2-3 \%$ concentration as discussed above. As also demonstrated in Figure 8(a) and 8(b), the contributions of ${ }^{3} \mathrm{He}$ populations to the CTS signal are below the alpha contribution but they are not negligible. The width of the region with non-negligible ${ }^{3} \mathrm{He}$ CTS signal fraction is $\sim 20-30 \mathrm{~cm}$ suggesting that at most two scattering volumes can be affected.

\section{Conclusions}

The fast ion CTS diagnostic will enable inferences about the fusion alpha distributions even in the presence of energetic ions due to off-axis ICRH in ITER. The triton CTS signal component is always at least an order of magnitude below the alpha CTS signal component, even in a scenario with an upgraded heating power of $40 \mathrm{MW}$. However, the strongest CTS signal contribution from ICRH is expected for a ${ }^{3} \mathrm{He}$ minority heating scheme. In a particular limited region, the contribution of the fast ${ }^{3} \mathrm{He}$ can be larger than $\sim 10 \%$ of the alpha feature, making it difficult to draw conclusions about the alpha particles for resolution of near perpendicular velocities. Outside this region with a width of $\sim 20-30 \mathrm{~cm}$, the results indicate that the CTS diagnostic will allow conclusions about physics of fast alpha particles.

\section{Acknowledgments}

This work, supported by the European Communities under the contract of Association between EURATOM and Ris $\varnothing$ DTU and CEA, was partly carried out within the framework of the European Fusion Development Agreement. The views and opinions expressed herein do not necessarily reflect those of the European Commission.

\section{References}

[1] ITER Physics Expert Group on Energetic Particles, Heating and Current Drive et al 1999 Nucl. Fusion 39 2471-2495

[2] Fasoli A, Gormenzano C, Berk H, Breizman B, Briguglio S, Darrow D, Gorelenkov N, Heidbrink W, Jaun A, Konovalov S, Nazikian R, Noterdaeme J M, Sharapov S, Shinohara K, Testa D, Tobita K, Todo Y, Vlad G and F Z 2007 Nucl. Fusion 47 S264 - S284

[3] Chen L and Zonca F 2007 Nucl. Fusion 47 S727 - S734

[4] Heidbrink W and Sadler G 1994 Nucl. Fusion 34535

[5] Heidbrink W 2002 Phys. Plasma 92213 - 2219

[6] Rosenbluth M and Rutherford P 1975 Phys. Rev. Lett. 34 1428-1431

[7] Cheng C and Chance M 1986 Phys. Fluids 29 3695-3701

[8] Wong K 1999 Plasma Phys. Control. Fusion 41 R1-R56

[9] Zweben S, Budny R, Darrow D, Medley S, Nazikian R, Stratton B, Synakowski E, Taylor G and the TFTR Group 2000 Nucl. Fusion 4091 - 149 
[10] Pinches S D, Berk H L, Borba D N, Breizman B N, Briguglio S, Fasoli A, Fogaccia G, Gryaznevich M P, Kiptily V, Mantsinen M J, Sharapov S E, Testa D, Vann R G L, Vlad G and Zonca F 2004 Plasma Phys. Control. Fusion 46 B187-B200

[11] Chen L 1994 Phys. Plasma 1 1519-1522

[12] Briguglio S, Fogaccia G, Vlad G, Zonca F, Shinohara K, Ishikawa M and Takechi M 2007 Phys. Plasma 14 055904-055904-10

[13] Bindslev H, Hoekzema J A, Egedal J, Fessey J A, Hughes T P and Machuzak J S 1999 Phys. Rev. Lett. 83(16) 3206-3209

[14] Bindslev H, Nielsen S K, Porte L, Hoekzema J A, Korsholm S B, Meo F, Michelsen P K, Michelsen S, Oosterbeek J, Tsakadze E L, Westerhof E, Woskow P and the TEXTOR team 2006 Phys. Rev. Lett. 97 205005-1-2005005-4

[15] Bindslev H, Nielsen S K, Porte L, Hoekzema J A, Korsholm S B, Meo F, Michelsen P K, Michelsen S, Oosterbeek J, Tsakadze E L, Westerhof E, Woskow P and the TEXTOR team 2007 Plasma Phys. Control. Fusion 49 B551-B562

[16] Nielsen S K, Bindslev H, Porte L, Hoekzema J A, Korsholm S B, Meo F, Michelsen P K, Michelsen S, Oosterbeek J, Tsakadze E L, Westerhof E, Woskow P and the TEXTOR team 2008 Phys. Rev. E $\mathbf{7 7} 16407$

[17] Meo F, Bindslev H, Korsholm S B, Furtula V, Leuterer F, Leipold F, Michelsen P K, Nielsen S K, Salewski M, Stober J, Wagner D and Woskov P 2008 Rev. Sci. Instrum. 79 10E729

[18] Bindslev H 1993 Plasma Phys. Control. Fusion 35(11) 1615-1640

[19] Bindslev H 1996 J. Atmos. Terr. Phys. 58983

[20] Egedal J, Bindslev H, Budney R and Woskov P 2005 Nucl. Fusion 45 191-200

[21] Salewski M, Asunta O, Eriksson L G, Bindslev H, Hynönen V, Korsholm S B, Kurki-Suonio T, Leipold F, Meo F, Michelsen P K, Nielsen S K and Roenby J 2009 Plasma Phys. Control. Fusion 51035006

[22] Costley A, Allen S, Andrew P, Bertalot L, Barnsley R, Duan X, Encheva A, Ingesson C, Johnson D, Lee H, Kawano Y, Krasilnikov A, Kumar V, Kusama Y, Marmar E, Pak S, Pitcher C, Rao C, Saibene G, Thomas D, Thomas P, Vasu P, Vayakis G, Walker C, Yang Q, Zaveriaev V and Zhao J 2008 Proceedings of the 22nd IAEA Conference (IAEA)

[23] Eriksson L G, Hellsten T and Willen U 1993 Nucl. Fusion 33 1037-48

[24] Eriksson L G and Hellsten T 1995 Phys. Scripta 5370

[25] Donne A, AE C, Barnsley R, Bindslev H, Boivin R, Conway G, Fisher R, Giannella R, Hartfuss H, von Hellermann M, Hodgson E, Ingesson L, Itami K, Johnson D, Kawano Y, Kondoh T, Krasilnikov A, Kusama Y, Litnovsky A, Lotte P, Nielsen P, Nishitani T, Orsitto F, Peterson B, Razdobarin G, Sanchez J, Sasao M, Sugie T, Vayakis G, Voitsenya V, Vukolov K, Walker C, Young K and the ITPA Topical Group on Diagnostics 2007 Nucl. Fusion 47 S337 - S384

[26] Bindslev H, Meo F, Tsakadze E, Korsholm S B and Woskov P 2004 Rev. Sci. Instrum. 75(10) $3598-3600$

[27] Meo F, Bindslev H, Korsholm S, Tsakadze E, Walker C, Woskov P and Vayakis G 2004 Rev. Sci. Instrum. 75 3585-3588

[28] Korsholm S B, Bindslev H, Furtula V, Leipold F, Meo F, Michelsen P K, Michelsen S, Salewski M and Tsakadze E L 2008 Burning Plasma Diagnostics (AIP conference proceedings vol 988) pp 118-122

[29] Salewski M, Meo F, Bindslev H, Furtula V, Korsholm S B, Lauritzen B, Leipold F, Michelsen P K, Nielsen S K and Nonbøl E 2008 Rev. Sci. Instrum. 79 10E729

[30] Shimada M, Campbell D, Mukhovatov V, Fujiwara M, Kirneva N, Lackner K, Nagami M, Pustovitov V, Uckan N, Wesley J, Asakura N, Costley A, Donné A, Doyle E, Fasoli A, Gormezano C, Gribov Y, Gruber O, Hender T, Houlberg W, Ide S, Kamada Y, Leonard A, Lipschultz B, Loarte A, Miyamoto K, Mukhovatov V, Osborne T, Polevoi A and Sips A 2007 Nucl. Fusion 47 S1-S17

[31] Polevoi A R, Medvedev S Y, Mukhovatov V S, Kukushkin A S, Murakami Y, Shimada M and 
Ivanov A A 2002 J. Plasma Fusion Res. SERIES 582

[32] Chang C 1985 Phys. Fluids 283598

[33] Bergeaud V, Eriksson L G and Start D 2000 Nucl. Fusion 40 35-51 